\title{
Cavernosopatía Traumática Aguda (CTA) trauma peneano en un grupo de 38 pacientes en un consultorio de medicina sexual durante 20 años de experiencia (1998-2018)
}

\section{Acute Traumatic Cavernosopathy Penile Trauma in a Group of 38 Patients in a Sexual Medicine Clinic during 20 Years of Experience (1998-2018)}

Juan Fernando Uribe A. ${ }^{1}$ ${ }^{1}$ Departamento de Urología, Hospital Pablo Tobón Uribe, Medellín,
Colombia

Urol Colomb 2019;28:307-312.
Address for correspondence Juan F. Uribe A., MD, Departamento de Urología, Hospital Pablo Tobón Uribe, Carrera 48 No 19 A 40 Of 1412, Medellín 050026, Colombia

(e-mail: medicinasexual@une.net.co; urologiahptu@gmail.com).

\section{Resumen}

\section{Palabras Clave}

- enfermedad peneana

- induración peneana

- traumas de pene

- fractura de pene

- disfunción eréctil

- fibrosis
Introducción El trauma de pene tiene una variedad de presentaciones, no siempre de origen sexual con diversas implicaciones anatómicas y funcionales para él.

Objetivo Describir los hallazgos en un grupo de pacientes de "Cavernosopatía traumática aguda" (CTA), desde el punto de vista clínico y ecográfico y sus desenlaces. Métodos Se realizó un estudio retrospectivo de un grupo de 38 pacientes que consultaron por CTA en un período de 20 años (1998-2018) en una consulta de medicina sexual en Medellín-Colombia. La condición para ser incluidos, era el antecedente en el tiempo de un trauma agudo y mayor del pene. Se evaluaron el tipo de trauma y variables relacionadas (crack audible, hematoma, fractura albugínea, ruptura del ligamento suspensorio, necesidad de cirugía); hallazgos clínicos (curvaturas, escotaduras, acortamiento); hallazgos ecográficos (Fibrosis peneana, fugas venosas) y otros desenlaces (disfunción eréctil). El estudio contó con la aprobación del comité de ética en investigación del HPTU.

Resultados Se estudiaron 38 pacientes con diagnóstico de CTA. Edad de consulta: 42 y edad del trauma 38,7 años, promedio. Trauma sexual (TS): 23 pacientes $(60,5 \%)$, No sexual (TNS): 15 pacientes (39,4\%). Hematomas: 22 (57,8\%); fractura albugínea: 16 (42,1\%); ruptura del ligamento suspensorio: 2 (5,2\%); curvatura secundaria: $14(36,8 \%)$; nódulos palpables: 13 (34,2\%); acortamiento: 11 (28,9\%); crack audible: 13 (34,2\%); algún grado de disfunción eréctil: 26 (68,4\%); necesidad de cirugía urgente: 6 (15,78\%). En los 31 (81,5\%) pacientes que tuvieron una ECO peneana como parte de su estudio tenemos, hallazgo de fibrosis: $25 / 31$ (80,6\%); fugas venosas cavernosas: $5 / 31(16,1 \%)$ e hipervascularización 10/31 (32,2\%). received

November 7, 2018

accepted

January 31, 2019
DOI https://doi.org/

10.1055/s-0039-1681085.

ISSN 0120-789X.

e ISSN 2027-0119.
Copyright ( 2019, Sociedad Colombiana License terms de Urología. Publicado por Thieme Revinter Publicações Ltda., Rio de Janeiro, Brazil. Todos los derechos reservados.

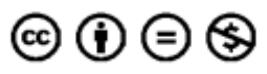




\begin{abstract}
Keywords

- penile disease

- penile induration

- penis trauma

- penis fracture

- erectile dysfunction

- fibrosis

Introduction Penis trauma has a variety of presentations, not always of sexual origin with various anatomical and functional implications for the penis.

ObjectiveTo describe the findings in a group of patients of "acute traumatic Cavernosophaty", from the clinical and echographics point of view and their outcomes. Methods A retrospective study of a group of 38 patients who consulted by CTA in a period of 20 years (1998-2018) in a sexual medicine clinic in Medellin, Colombia, was conducted. The condition to be included was the antecedent in time of an acute and major trauma of the penis. The type of trauma and related variables were evaluated (audible crack, hematoma, albuginea fracture, rupture of the suspensory ligament, need for surgery); clinical findings (curvatures, notches, shortening); ultrasound findings (penile fibrosis, venous leaks) and other outcomes (erectile dysfunction). The study was approved by the research ethics committee of the HPTU.

Results Thirty-eight patients with diagnosis of CTA were studied. Age of consultation: 42 and age of the trauma 38.7 years, average. Sexual trauma (TS): 23 patients (60.5\%), Nonsexual (TNS): 15 patients (39.4\%). Haematoma: 22 (57.8\%); albugineal fracture: 16 (42.1\%); rupture of the suspensory ligament: 2 (5.2\%); secondary curvature: 14 (36.8\%); palpable nodules: 13 (34.2\%); shortening: 11 (28.9\%); audible crack: 13 (34.2\%); some degree of erectile dysfunction: 26 (68.4\%); need for urgent surgery: 6 (15.78\%). In the 31 (81.5\%) patients who had a penile ECO as part of their study: fibrosis finding: $25 / 31$ (80.6\%); venous cavernous leaks: $5 / 31$ (16.1\%) and hypervascularization 10/31 (32.2\%).

Conclusions Acute traumatic cavernosopathy is an appropriate name for the syndrome that triggers a greater and acute penile trauma, which can include sequelae such as penile fibrosis (up to $80 \%$ ), secondary curvatures (up to $38 \%$ ), shortening, venous leaks and erectile dysfunction (up to $70 \%$ of cases). $6 / 10$ of these traumas are of a sexual nature and the rest are a motley group of traumas (car, sports, burns, animal attacks).
\end{abstract}

Conclusiones La Cavernosopatía traumática aguda (CTA) es una denominación adecuada para el síndrome que desencadena un trauma peneano agudo, que puede incluir secuelas como fibrosis peneana (hasta el $80 \%$ ), curvaturas secundarias (hasta el $38 \%$ ), acortamiento, fugas venosas y disfunción eréctil (hasta $70 \%$ de los casos). Entre $6 / 10$ de esos traumas, son de índole sexual y el resto son un grupo variopinto de traumas (automovilísticos, deportivos, quemaduras, ataques de animales).

\section{Introducción}

El trauma de pene tiene una variedad de presentaciones y generan diversas formas de clasificarlo. Un primer tipo de clasificación puede considerar el origen del trauma; otra forma de clasificación divide el trauma según la etiología, según si su origen es sexual o pertenece a un grupo variopinto de origen no sexual. Para Morey y col., ${ }^{1}$ el trauma fue definido como una "injuria causada por una fuerza externa desde una variedad de mecanismos, incluyendo traumas relacionados al tráfico o el transporte, caídas, asaltos (ej. armas contundentes, apuñalamientos, disparos), explosiones y otras tantas más..." La literatura considera que la fractura de pene es una emergencia urológica rara, que ocurre en 1 de cada 175,000 admisiones a urgencias, con sitios del mundo donde existen cifras descomunales de eventos relacionados más con la idiosincrasia religiosa (uso del Taghaandan o bloqueo de la erección). ${ }^{2,3}$ El trauma sexual a diferencia de los otros, tiene como característica fundamental que el pene se encuentra en erección, usualmente es precedido por una actividad sexual agresiva, aunque también puede ocurrir durante masturbación, durante posiciones inusuales o fuerzas que doblan el pene. Pueden sub yacer, además de las fuerzas excesivas durante el coito, uretritis crónica o daños de la túnica albugínea como factores de riesgo. En el cuadro clínico habitual, el paciente a menudo escucha un crack, seguido de una rápida detumescencia, dolor, a veces severo, edema subcutáneo, equimosis o hematomas de tamaño variable. El pene puede tener deformidades. Los traumas no sexuales en cambio, son un grupo variopinto, con heridas penetrantes o no al cuerpo cavernoso y pueden incluir causas tan disímiles como accidentes de tránsito, deportivos, golpes directos, heridas de PAF, heridas con armas cortantes, explosiones, mordeduras y picaduras de animales venenosos. La literatura habla de 
situaciones tan extrañas como ruptura de una enfermedad de Mondor ${ }^{4}$ o la ruptura de la vena dorsal superficial que semeja una fractura peneana. ${ }^{5}$ También de mordeduras e amputaciones autoinfligidas (Síndrome de Klignsor). ${ }^{6}$ Existe un grupo que podría considerarse intermedio entre lo sexual y lo no sexual, que es el trauma por el uso de aparatos constrictivos de diversa índole, que suelen producir daños en los tejidos blandos y que no fueron incluidos en esta revisión. Las complicaciones habitualmente están relacionadas al daño de la túnica albugínea no reparada: cicatriz fibrosa (a menudo mal llamada enfermedad de Peyronie), erecciones dolorosas, disfunción eréctil y a veces, estrechez de la uretra. Según Hatzichristodoulou y col., ${ }^{7}$ la disfunción eréctil, aun con manejo quirúrgico, es tan alto como $60 \%$ o más. La función eréctil debería ser evaluada, además de algún puntaje validado, con una ecografía dopler del pene, que idealmente debería ser pre y post quirúrgico, aunque su uso rutinario puede considerarse controversial, lo mismo que otros métodos diagnósticos como el TAC o la resonancia magnética nuclear. ${ }^{8-18}$ El objetivo del presente estudio fue describir los hallazgos en un grupo de pacientes de Cavernosopatía traumática aguda (CTA), desde el punto de vista clínico y ecográfico y sus desenlaces.

\section{Métodos}

Se realizó un estudio retrospectivo de un grupo de 38 pacientes que consultaron por un trauma peneano agudo (CTA), en un período de 20 años (1998-2018), en una consulta de medicina sexual y servicio de ecografía peneana en Medellín-Colombia. La condición para ser incluidos era el antecedente de un trauma agudo y significativo en el pene, independiente del tiempo de evolución. Fueron excluidos los pacientes de trauma de tejidos blandos simples, traumas por inyecciones intracavernosas, procedimientos de priapismo y en general todos los post quirúrgicos. Se evaluaron los hallazgos clínicos del trauma (origen, crack, hematoma, fractura del cavernoso, ruptura del ligamento suspensorio, necesidad de cirugía); hallazgos clínicos (curvaturas, escotaduras, acortamiento); se verificaron la presencia o no de curvaturas peneanas, dividiéndola en primarias (desde el comienzo de la vida sexual) y secundarias (que aparecieron luego en la vida sexual). Se evaluaron los hallazgos ecográficos en 31 pacientes (81\%) que tuvieron en su evaluación una Ecografía dopler high definition de pene con vasoactivo (usando 0,1 cc de prostaglandina E1 o de mezcla de prostaglandina, papaverina y fentolamina - Trimix), por el mismo examinador usando el mismo equipo (Voluson E8 General Electric). ${ }^{19}$ En ese grupo específico se cuantificó la fibrosis usando la clasificación ecográfica del grado de fibrosis de Laurence Levine (grado 1-Leve, grado 2-Moderada, grado 3 Grave) Las áreas de calcificación fueron definidas como regiones hiperecoicas con la presencia de sombra acústica usando los tres grados ecográficos propuestos por Levine y sus colaboradores: Grado 3, (placas $>1,5 \mathrm{~cm}$ en cualquier dimensión o múltiples placas $\geq 1,0 \mathrm{~cm}$ ); grado 2 (lesiones de $0,3 \mathrm{mma} 1,5 \mathrm{~cm}$ ) y grado 1 (lesiones $<0,3 \mathrm{~mm}$ ). ${ }^{11,18,20-23}$ Se evaluó la presencia de fugas venosas dorsales o cavernosas que se eco localizaron; el otro desenlace evaluado fue la disfunción eréctil; los pacientes tenían una pesquisa sobre disfunción eréctil, considerándose disfuncional con cualquiera de estas dos escalas: un puntaje menor a 21 puntos en la escala del SHIM (Sexual Health inventory for men) o si habían respondido negativamente a la pregunta sobre calidad de la erección en las últimas 4 semanas según la "Erection Hardness Score" (EHS). En el análisis estadístico se incluyó el universo de historias clínicas de los pacientes que cumplieron los criterios de inclusión y exclusión. El resumen de las variables cualitativas se presenta con número absoluto y porcentaje, la edad se presenta con media y desviación estándar. La fibrosis como factor de exposición se dividió en tres categorías (leve, moderada, grave) y se evaluó la asociación con los otros factores clínicos y ecográficos. El estudio contó con la aprobación escrita del comité de ética en investigación del Hospital Pablo Tobón Uribe y quedó inscrito dentro de los trabajos aprobados de esa institución.

\section{Resultados}

Se estudiaron 38 pacientes con diagnóstico de CTA. La Edad de consulta fue de 42 años y la edad del trauma de 38,7 años, en promedio para el grupo en general. Cuando se realizó la comparación de edad del trauma para los dos grupos principales, se encontró que el promedio en el trauma sexual (TS) fue de 45,2 años y en el trauma no sexual (TNS) fue de 28 años.

El grupo de trauma sexual (TS) estaba constituido por 23 pacientes (60,5\%); en ese grupo 22 tuvieron un trauma coital y 1, trauma durante maniobras masturbatorias. El trauma No sexual (TNS) involucró a 15 pacientes (39,47\%), cuya etiología era variopinta: 5 accidentes de tránsito (13,1\%), 3 quemaduras severas (7,9\%), 2 traumas deportivos (5,2\%), una herida de PAF $(2,6 \%), 2$ traumas directos (5,2\%), un trauma auto infligido $(2,6 \%)$ y una picadura en genitales de una araña Loxosceles Laeta (2,6\%). Esos dos últimos pacientes merecen un comentario adicional: El paciente de la maniobra auto infligida, describió un típico Taghaandan para bloquear una erección en público, por asuntos de pudor, no por motivos religiosos, demostrando lo peligroso de ese mecanismo que en tantos pacientes genera en ciertas comunidades, en donde la erección en sitios sagrados puede considerarse un pecado grave. El caso del paciente con el cuadro de Laxoscelismo visceral y cutáneo, fue ampliamente estudiado por la empresa de riesgos laborales del paciente, al ser un accidente de trabajo, en donde incluso pudo capturarse la araña y ubicar detalladamente su procedencia. (-Tabla 1 y - Fig. 1).

Con respecto a los hallazgos clínicos, encontramos lo siguiente: Crack audible: TS 11 (56,5\%) vs TNS (0\%); fractura albugínea: TS 12 (69\%) vs TNS (0\%); curvatura secundaria: TS $7(30,4 \%)$ vs TNS 7 (46,6\%), TS + TNS: 14 (36,8\%); nódulos palpables: TS 7 (30,4\%) vs TNS 6 (40\%), TS + TNS: 13 (34,2\%); Hematomas: TS $15(65,2 \%)$ vs TNS 7 (46,6\%), TS + TNS: 22 (57,8\%); acortamiento: TS 8 (34,7\%) vs TNS 3 (20\%), TS + TNS: $11(28,9 \%)$; ruptura del ligamento suspensorio: TS 1 (4,3\%) vs TNS 1 (6,6\%), TS + TNS: 2 (5,2\%); algún grado de disfunción eréctil secundaria: TS 16 (70\%) vs 
Tabla 1 Clasificación según el tipo de trauma

\begin{tabular}{|c|c|c|c|c|}
\hline \multicolumn{3}{|l|}{ Tipo de trauma } & N. de pacientes & Porcentaje (\%) \\
\hline \multicolumn{3}{|l|}{ Trauma sexual } & 23 & 60,52 \\
\hline \multirow{9}{*}{$\begin{array}{l}\text { Trauma no sexual } \\
\text { (n: } 15-39,47 \%)\end{array}$} & \multicolumn{2}{|l|}{ Autoinfligido (Taghaandan) } & 1 & 2,63 \\
\hline & \multicolumn{2}{|l|}{ Accidentes de tránsito } & 5 & 13,15 \\
\hline & \multirow[t]{3}{*}{ Quemaduras (n: 3 - 7,89\%) } & Aceite & 1 & 2,63 \\
\hline & & Eléctrica & 1 & 2,63 \\
\hline & & Hielo & 1 & 2,63 \\
\hline & \multicolumn{2}{|l|}{ Trauma deportivo } & 2 & 5,26 \\
\hline & \multicolumn{2}{|l|}{ Herida de PAF } & 1 & 2,63 \\
\hline & \multicolumn{2}{|l|}{ Trauma directo por caída } & 2 & 5,26 \\
\hline & \multicolumn{2}{|c|}{ Mordedura de araña (Loxosceles Laeta) } & 1 & 2,63 \\
\hline \multicolumn{3}{|l|}{ TOTAL } & 38 & 100 \\
\hline
\end{tabular}

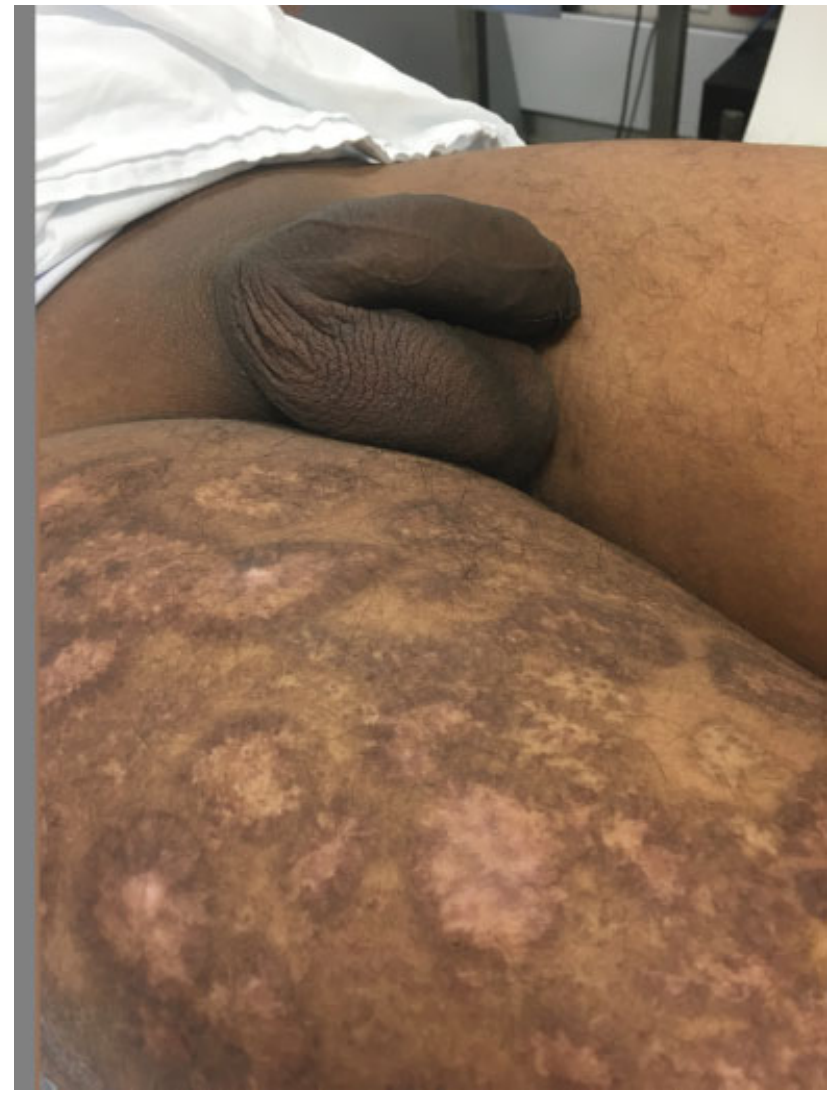

Fig. 1 Laxoscelismo genital por picadura de araña Loxosceles Laeta.

TNS 10 (66,6\%), TS + TNS: 26 (68,4\%); necesidad de cirugía urgente: TS $4(17,3 \%)$ vs TNS 2 (13,3\%), TS + TNS: 6 $(15,8 \%)^{8,24}$ (- Tabla 2)

En los 31/38 pacientes que fueron sometidos a ECO doppler de pene encontramos lo siguiente: Con ECO realizada: TS $17 / 23(73,9 \%)$, TNS $14 / 15(93,3 \%)$, TS + TNS: $31(81,5 \%)$; fibrosis cavernosa: TS $14(83,2 \%)$, TNS 11 (78,5\%), TS + TNS: 25 (80,6\%); fibrosis leve Levine 1: 2(8\%), fibrosis moderada Levine 2: 19 (76\%) y Fibrosis severa Levine 3: 4 (16\%); fugas venosas cavernosas: TS 1 (5,88\%), TNS 4
(23,5\%), TS + TNS: 5 (16,1\%); fugas dorsales: TS 2 (11,76\%), TNS 3(21,42\%), TS + TNS: $5 \quad(16,12 \%)$; hipervascularización: TS 6 (35,2\%), TNS 4(28,57\%), TS + TNS: $10(32,25 \%){ }^{25,26}$ (- Tabla 3).

\section{Discusión}

Con respecto a la edad, cuando se realizó la comparación de edad del trauma para los dos grupos principales, se encontró que el promedio en el trauma sexual (TS) fue de 45,2 años y en el trauma no sexual (TNS) fue de 28 años. Esos datos están de acuerdo con los hallazgos habituales de la literatura, puesto que se menciona la cuarta y quinta décadas de la vida como las de mayor frecuencia de eventos sexuales. El grupo variopinto de los no sexuales (TNS), ocurrió en pacientes más jóvenes, lo que corresponde adecuadamente con la etiología de los traumas (deportivos, automovilísticos, etc). ${ }^{3}$

Otros estudios que analizan el tema de fracturas de pene, encuentran una frecuencia de hematomas del 98\% y del crack audible del $70 \%{ }^{2}$ En el presente estudio, el hallazgo de hematomas en trauma sexual (TS) fue de un 65\% y de crack audible fue del $56 \%$, lo que se explica porque en ese grupo, solamente el $60 \%$ cursaron con fractura (ruptura de la albugínea) y el grupo incluía traumas sexuales significativos, aunque sin fractura.

Con respecto a la necesidad de una cirugía ulterior al trauma, los datos deben analizarse así: En el grupo más comprometido de pacientes, con un trauma sexual, presencia de hematoma, evidencia de ruptura de fascia (16 pacientes), apenas se hicieron 4 cirugías y en el grupo completo de 38 pacientes, 6 cirugías. Esos datos contradicen las recomendaciones de las publicaciones especializadas sobre el tema del manejo de esas urgencias. ${ }^{7,8}$

En el uso de algún método diagnóstico para evaluar el trauma, encontramos que el $80 \%$ de los pacientes fueron sometidos a una ECO doppler de pene con uso de vasoactivo. Sin embargo, tiende a utilizarse tardíamente para evaluar secuelas y no en el momento agudo para definir conductas. ${ }^{21}$ 
Tabla 2 Hallazgos clínicos según tipo de trauma

\begin{tabular}{|c|c|c|c|c|c|}
\hline \multirow[t]{2}{*}{ Hallazgos clínicos } & \multicolumn{2}{|c|}{$\begin{array}{l}\text { Trauma sexual } \\
(\mathrm{n}: 23)\end{array}$} & \multicolumn{2}{|c|}{$\begin{array}{l}\text { Trauma no sexual } \\
\text { (n:15) }\end{array}$} & \multirow{2}{*}{$\begin{array}{l}\text { TOTAL } \\
\text { n:38 } \\
\text { n (\%) }\end{array}$} \\
\hline & $\mathrm{n}$ & $\%$ & $n$ & $\%$ & \\
\hline Crack audible & $13 / 23$ & 56,52 & 0 & 0 & $13 / 38(34,21)$ \\
\hline Fractura albugínea & $16 / 23$ & 69,56 & 0 & 0 & $16 / 38(42,10)$ \\
\hline Curvatura primaria & 0 & 0 & $4 / 15$ & 26,66 & $4 / 38(10,52)$ \\
\hline Curvatura secundaria & $7 / 23$ & 30,43 & $7 / 15$ & 46,66 & $14 / 38(36,84)$ \\
\hline Nódulo palpable & $7 / 23$ & 30,43 & $6 / 15$ & 40 & $13 / 38(34,21)$ \\
\hline Hematoma & $15 / 23$ & 65,21 & $7 / 15$ & 46,66 & $22 / 38(57,89)$ \\
\hline Acortamiento & $8 / 23$ & 34,78 & $3 / 15$ & 20 & $11 / 38(28,94)$ \\
\hline Ruptura ligamento suspensorio & $1 / 23$ & 4,34 & $1 / 15$ & 6,66 & $2 / 38(5,26)$ \\
\hline Disfunción Eréctil & $16 / 23$ & 69,56 & $10 / 15$ & 66,66 & $26 / 38(68,42)$ \\
\hline Cirugía & $4 / 23$ & 17,39 & $2 / 15$ & 13,33 & $6 / 38(15,78)$ \\
\hline
\end{tabular}

Tabla 3 Hallazgos ecográficos según el tipo de trauma

\begin{tabular}{|c|c|c|c|c|c|}
\hline \multirow[t]{2}{*}{ Hallazgos ecográficos } & \multicolumn{2}{|c|}{$\begin{array}{l}\text { Trauma Sexual } \\
\text { (con ECO) } \\
\text { (n: 17/23) }\end{array}$} & \multicolumn{2}{|c|}{$\begin{array}{l}\text { Trauma No sexual } \\
\text { (con ECO) } \\
\text { (n: } 14 / 15)\end{array}$} & \multirow[t]{2}{*}{$\begin{array}{l}\text { TOTAL } \\
\mathrm{n}: 31 \\
\mathrm{n}(\%)\end{array}$} \\
\hline & $\mathrm{n}$ & $\%$ & $\mathrm{n}$ & $\%$ & \\
\hline Fibrosis cavernosa & $14 / 17$ & 82,35 & $11 / 14$ & 78,57 & $25 / 31(80,64)$ \\
\hline Fuga dorsal & $2 / 17$ & 11,76 & $3 / 14$ & 21,42 & $5 / 31(16,12)$ \\
\hline Fuga cavernosa & $1 / 17$ & 5,88 & $4 / 14$ & 28,57 & $5 / 31(16,12)$ \\
\hline Hipervascularización & $6 / 17$ & 35,29 & $4 / 14$ & 28,57 & $10 / 31(32,25)$ \\
\hline
\end{tabular}

El hallazgo de un 70\% de pacientes con algún grado de disfunción eréctil, debe alertar a los interesados, en las consecuencias, a veces tardías, que un trauma puede tener en el correcto funcionamiento de la hemodinámica peneana. ${ }^{2}$

\section{Conclusiones}

La Cavernosopatía traumática aguda (CTA) es una denominación adecuada para el síndrome que desencadena en el pene, un trauma agudo, pudiendo incluir secuelas como fibrosis peneana (hasta el 80\%), curvaturas secundarias (hasta el 38\%), acortamiento, fugas venosas y disfunción eréctil secundaria (hasta en el 70\% de los casos). Entre 6 y 10 de esos traumas fueron de índole sexual y el resto de un grupo variopinto (Traumas automovilísticos, deportivos, quemaduras, insectos o animales). El promedio de edad muestra que el fenómeno ocurre predominantemente en jóvenes (38,7 años) en especial los no sexuales (28 años en promedio). A pesar del número de fracturas albugíneas (hasta el 60\% en los traumas de origen sexual), solo $16 \%$ van a cirugía, contradiciendo las recomendaciones habituales de diferentes artículos.

\section{Responsabilidades Éticas}

Protección de personas y animales. Los autores declaran que para esta investigación no se han realizado experimentos en seres humanos ni en animales.
Confidencialidad de los datos. Los autores declaran que en este artículo no aparecen datos de pacientes.

\section{Derecho a la privacidad y al consentimiento informado.}

Los autores declaran que en este artículo no aparecen datos de pacientes.

Financiación

Ninguna.

Conflicto de intereses

Los autores declaran no tener ningún conflicto de intereses.

\section{Referencias}

1 Morey AF, Brandes S, Dugi DD III, et al; American Urological Assocation. Urotrauma: AUA guideline. J Urol 2014;192(02):327-335

2 Falcone M, Garaffa G, Castiglione F, Ralph DJ. Current Management of Penile Fracture: An Up-to-Date Systematic Review. Sex Med Rev 2018;6(02):253-260

3 Agrawal SK, Morgan BE, Shafique M, Shazely M. Experience with penile fractures in Saudi Arabia. Br J Urol 1991;67(06): 644-646

4 Ganem JP, Kennelly MJ. Ruptured Mondor's disease of the penis mimicking penile fracture. J Urol 1998;159(04):1302

5 Bar-Yosef Y, Greenstein A, Beri A, Lidawi G, Matzkin H, Chen J. Dorsal vein injuries observed during penile exploration for suspected penile fracture. J Sex Med 2007;4(4 Pt 2): $1142-1146$ 
6 Aggarwal G, Adhikary SD. Klingsor syndrome: A rare surgical emergency. Ulus Travma Acil Cerrahi Derg 2017;23(05):427-429. Doi: $10.5505 /$ tjtes.2017.30346

7 Hatzichristodoulou G, Dorstewitz A, Gschwend JE, Herkommer K, Zantl N. Surgical management of penile fracture and long-term outcome on erectile function and voiding. J Sex Med 2013;10(05): $1424-1430$

8 Gaspar SS, Dias JS, Martins F, Lopes TM. Sexual Urological Emergencies. Sex Med Rev 2015;3(02):93-100

9 Miller S, McAninch JW. Penile fracture and soft tissue injury. Chapter 59. In: McAninch JW, ed. Traumatic and reconstructive urology. Philadelphia, PA: W.B. Saunders; 1996:693-698

10 El-Bahnasawy MS, Gomha MA. Penile fractures: the successful outcome of immediate surgical intervention. Int J Impot Res 2000; 12(05):273-277

11 Kramer AC. Penile fracture seems more likely during sex under stressful situations. J Sex Med 2011;8(12):3414-3417

12 Al-Shaiji TF, Amann J, Brock GB. Fractured penis: diagnosis and management. J Sex Med 2009;6(12):3231-3240, quiz 3241

13 Morey AF, Rozanski TA. Genital and lower urinary tract trauma. Chapter 83. In: Wein A, Kavoussi L, Novick A, Partin A, Peters C, eds. Campbell-Walsh urology. 9th edition. Philadelphia, PA: Saunders Elsevier; 2007:2649-2662

14 Zargooshi J. Trauma as the cause of Peyronie's disease: penile fracture as a model of trauma. J Urol 2004;172(01):186-188

15 Ibrahiem HI, el-Tholoth HS, Mohsen T, Hekal IA, el-Assmy A. Penile fracture: long-term outcome of immediate surgical intervention. Urology 2010;75(01):108-111

16 Karadeniz T, Topsakal M, Ariman A, Erton H, Basak D. Penile fracture: differential diagnosis, management and outcome. $\mathrm{Br} \mathrm{J}$ Urol 1996;77(02):279-281
17 Buyukkaya R, Buyukkaya A, Ozturk B, Kayıkçı A, Yazgan Ö. Role of ultrasonography with color-Doppler in the emergency diagnosis of acute penile fracture: a case report. Med Ultrason 2014;16(01): 67-69

18 Kuribayashi S, Takao T, Yamamichi G, et al. [Two Cases of Penile Fracture Diagnosed by Magnetic Resonance Imaging]. Hinyokika Kiyo 2016;62(09):501-503

19 Berookhim BM. Doppler Duplex Ultrasonography of the Penis. J Sex Med 2016;13(04):726-731

20 Levine LA, Coogan CL. Penile vascular assessment using color duplex sonography in men with Peyronie's disease. J Urol 1996; 155(04):1270-1273

21 Levine LA, Greenfield JM. Establishing a standardized evaluation of the man with Peyronie's disease. Int J Impot Res 2003;15 (Suppl 5):S103-S112

22 Yuruk E, Serefoglu EC. Re: Peyronie's disease plaque calcificationprevalence, time to identification, and development of a new grading classification. J Sex Med 2014;11(05):1351

23 Bekos A, Arvaniti M, Hatzimouratidis K, Moysidis K, Tzortzis V, Hatzichristou D. The natural history of Peyronie's disease: an ultrasonography-based study. Eur Urol 2008;53(03):644-650

24 Alnadhari I, Abdelhaleem Abdeljaleel O, Sampige VRP, Abdulmuhsin A, Shamsodini A. Penile Fracture: Simultaneous Complete Urethral Rupture with Bilateral Corpora Cavernosa Rupture. Case Rep Urol 2018;2018:4929518

25 Hassali MA, Nouri AI, Hamzah AA, Verma AK. Role of Penile Doppler as a Diagnostic Tool in Penile Fracture. J Med Ultrasound 2018;26(01):48-51. Doi: 10.4103/JMU.JMU_9_18

26 Nizamani WM, Ali SI, Vaswani AK, Shahani BK. Ultrasound Diagnosis of Penile Fracture. J Coll Physicians Surg Pak 2015;25 (Suppl 2):S12-S13. Doi: 10.2015/JCPSP.S84S85 